\title{
New Models of Emergency Prehospital Care That Avoid Unnecessary Conveyance to Emergency Department: Translation of Research Evidence into Practice?
}

\author{
Helen Anne Snooks, Mark Rhys Kingston, \\ Rebecca Elizabeth Anthony, and Ian Trevor Russell
}

Centre for Health Information Research and Evaluation (CHIRAL), Institute of Life Science, College of Medicine, Swansea University, Singleton Park, Swansea SA2 8PP, UK

Correspondence should be addressed to Helen Anne Snooks; h.a.snooks@swansea.ac.uk

Received 21 December 2012; Accepted 15 April 2013

Academic Editors: S. Huckson, E. Lang, and B. Rowe

Copyright (C) 2013 Helen Anne Snooks et al. This is an open access article distributed under the Creative Commons Attribution License, which permits unrestricted use, distribution, and reproduction in any medium, provided the original work is properly cited.

Background. Achieving knowledge translation in healthcare is growing in importance but methods to capture impact of research are not well developed. We present an attempt to capture impact of a programme of research in prehospital emergency care, aiming to inform the development of EMS models of care that avoid, when appropriate, conveyance of patients to hospital for immediate care. Methods. We describe the programme and its dissemination, present examples of its influence on policy and practice, internationally, and analyse routine UK statistics to determine whether conveyance practice has changed. Results. The programme comprises eight research studies, to a value of $>£ 4 \mathrm{~m}$. Findings have been disseminated through 18 published papers, cited 274 times in academic journals. We describe examples of how evidence has been put into practice, including new models of care in Canada and Australia. Routine statistics in England show that, alongside rising demand, conveyance rates have fallen from $90 \%$ to $58 \%$ over a 12 -year period, 2,721 million fewer journeys, with publication of key studies 2003-2008. Comment. We have set out the rationale, key features, and impact on practice of a programme of publicly funded research. We describe evidence of knowledge translation, whilst recognising limitations in methods for capturing impact.

\section{Background}

The gap between the production of research evidence and implementation into routine clinical practice has been well acknowledged and has been referred to as the second translational gap; the first gap is that between laboratory science and clinical research [1]. With increasing recognition of the importance of not only carrying out research but also of ensuring that research findings are taken up and used by those making health care policy and providing health care, researchers and research funders are now paying more attention to dissemination, particularly active forms which have the ability to influence care delivery, and also to capturing the impact of research [2-4].

In the field of emergency care, research evidence to underpin care has been criticised both for its scarcity and quality [5]. In the prehospital setting these concerns are even more acute [6-8]. Emergency prehospital care is a field without a strong academic tradition, but patient volume is high and outcomes are linked to responses provided by emergency medical systems (EMS). In this growing field, demonstrating impact in practice is fundamental to the continued attraction of research funding, building of research skills and culture, and thus a high-quality evidence based to inform future policy and practice.

With sustained increases in demand for emergency prehospital care across the developed world, current systems are unable to maintain services that traditionally respond to all emergency calls to the ambulance service with a paramedic staffed patient carrying vehicle travelling on lights and sirens, and with a default of conveyance to an emergency department (ED) for medical care unless the patient refused to travel. 
Researches focussing on the needs and outcomes of patients for whom emergency (999) calls to the ambulance service are made have shown that a substantial proportion of these patients (up to $52 \%$ ) $[11,12,15,28]$ do not need immediate medical care, but that triage systems at the despatch centre and on scene that identify patients for self- or communitybased care carry significant safety risks [15-17, 22]. Unnecessary transportation can also be an issue for patients who have little or no chance of survival [29].

In this paper we describe a programme of research related to the development and implementation of new models of care that allow ambulance services to offer alternatives to the traditional response and to safely reduce conveyance of patients to ED and present data that demonstrate the impact of this research.

\section{Summary of Research Programme}

Supported by over $£ 4 \mathrm{~m}$ in research grants, the programme of work includes studies that have followed the UK's Medical Research Council's Framework for the Development and Evaluation of Complex Interventions [30, 31] and comprises reviews of existing research and practice and experimental research, including randomised controlled trials (Table 1). Research findings indicate and describe the problem [9-13] and then the costs and effectiveness of alternatives to current practice $[14-19,22]$. Study findings have been widely disseminated to generic and specialist audiences through publication in peer reviewed and practitioner journals, as well as at conferences at local, national, and international levels. The research team works closely with prehospital care providers and policy makers in the UK but does not follow a formal knowledge transfer strategic approach.

\section{Impact on Policy and Practice: Knowledge Transfer}

3.1. Methods. We tracked citations using Google Scholar, undertook extensive electronic searching of policy documents, and gathered ad hoc information related to service developments in which studies from this programme of work were cited. We analysed routine national data provided by all individual services as part of their required performance statistics for the period before and since publication of findings from studies within the programme.

3.2. Results. Papers reported in Table 1 have been cited in academic journals 274 times to date. An influential systematic review of 999 alternatives for the UK Department of Health (2005) draws heavily upon the work of the research team and has gone on to influence guidance emanating from statutory UK bodies [32-34]. Nontransport (to ED) guidelines from the Ambulance Service Association and Department of Health, which cite elements of this work, have been widely adopted, as have "Treat and Refer" protocols.

Enhanced telephone triage has been adopted across the UK ambulance service providers, in line with the recommendations of the Department of Health and the Ambulance
Service Association-both of which respond to work published within this programme. Through correspondence and desktop reviews we are aware of similar service models having been adopted internationally, in Canada, for example, and in South Australia, where the Ambulance Service was able to report financial savings following implementation across the state of Victoria, having cited findings from the Telephone Advice Study [13-15], in their business case.

"Prior to 2003 we sent an ambulance to all calls received via the "000" ambulance emergency call centre. Professor Snooks work, including the evidence of very high caller satisfaction and very few adverse events from referrals instead of conveyance, was used to show the need for a referral service at point of call. In the year following implementation we were able to show cost savings and have now fully implemented the service, and the model is being rolled out across Australia." Senior Research Fellow, Ambulance Victoria, Australia.

"We have used Professor Snooks published work [on pre hospital clinical decision making tools] to inform policies in Nova Scotia and Alberta. There are similar challenges being faced in the UK and Canada." Senior Performance Strategist, Alberta Health Services Emergency Medical Services.

Evidence of the impact of the work in prehospital care can be seen in conveyance rates-90\% of emergency calls in England resulted in hospital conveyance in 2000 compared to $58 \%$ in 2012 (see Figures 1 and 2)-equivalent to 2,721 million fewer journeys.

\section{Discussion}

4.1. Key Points. Findings from this programme of work have consistently highlighted the need for alternatives to routine conveyance of 999 patients to ED and the team have developed, implemented, and tested a range of approaches to improving and providing evidence about the quality, safety, and cost effectiveness of care.

Working collaboratively with the NHS and policy makers, lessons from the programme of work have been disseminated widely in peer-reviewed articles, policy literature, international conferences, and through personal invitations to visit service providers.

Nationally and internationally, evidence from this programme of work has been cited in policy documents and in service developments, including the provision of telephone advice and Treat and Refer protocols.

In the face of consistent increases in demand for the 999 emergency ambulance service in the UK and internationally, we have demonstrated evidence of falling conveyance rates and an increasing proportion of patients treated at scene in England since the publication of our findings.

4.2. Strengths and Limitations. Methods for capturing impact of research are not well developed and include a variety of 


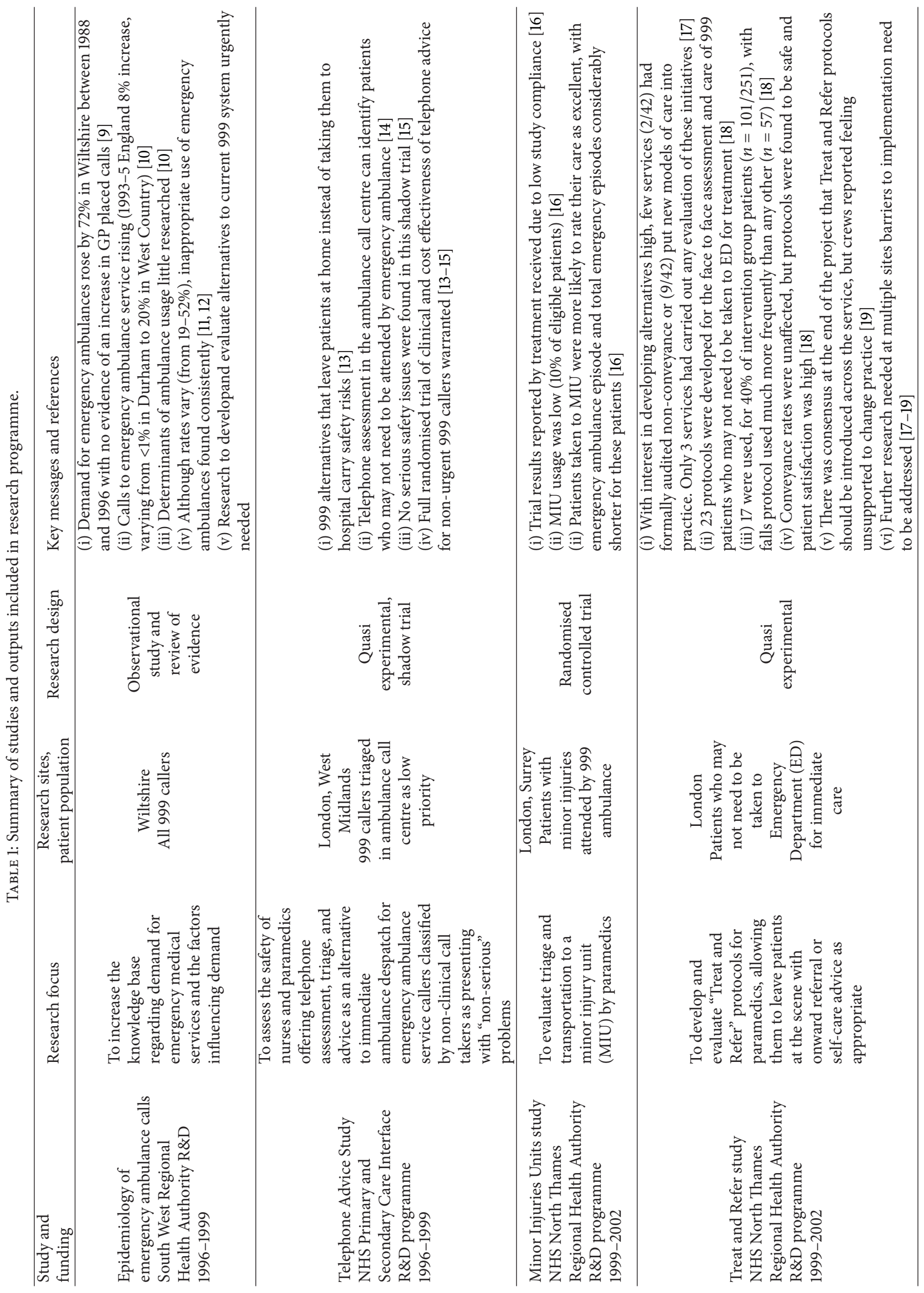




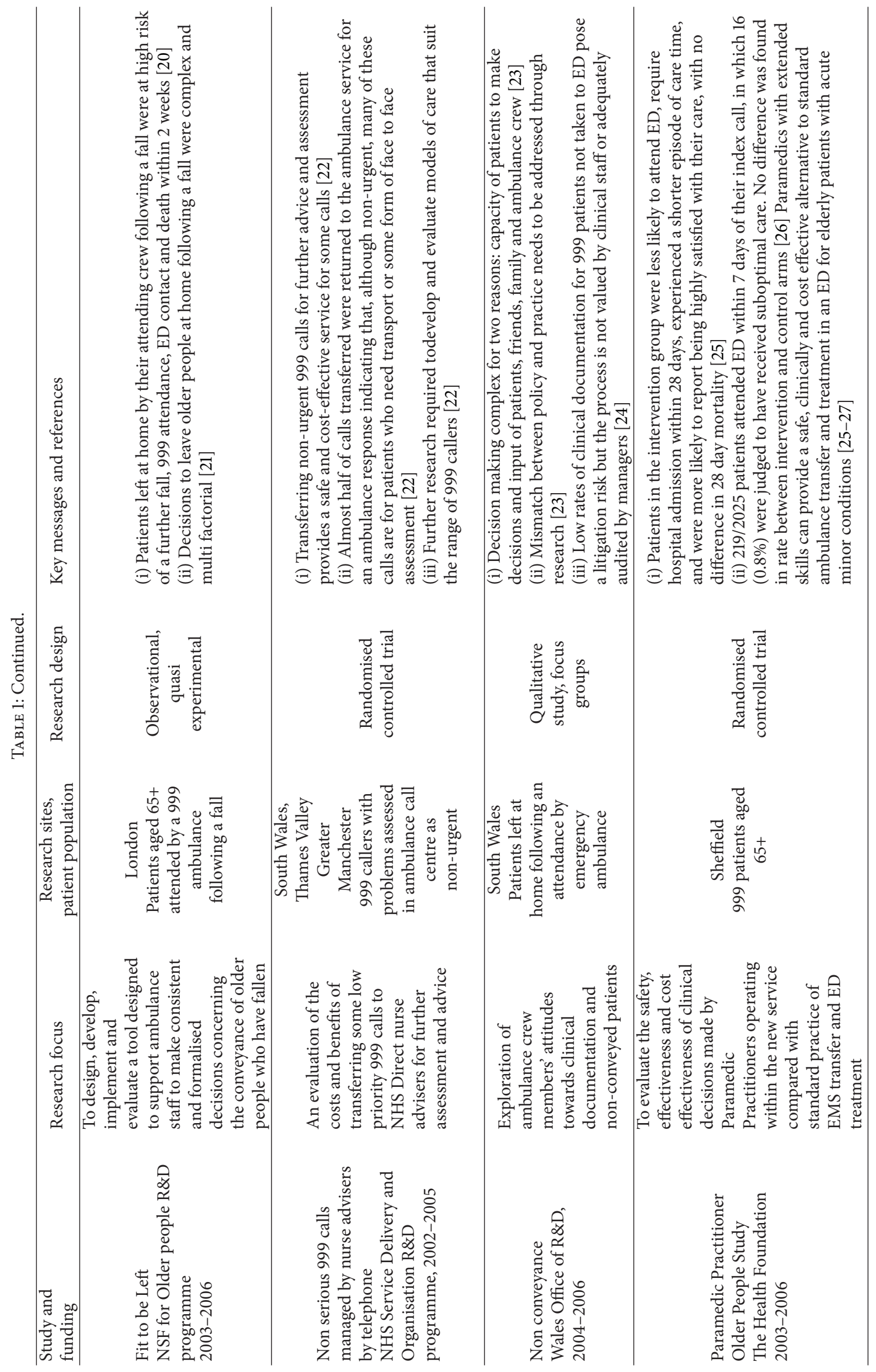




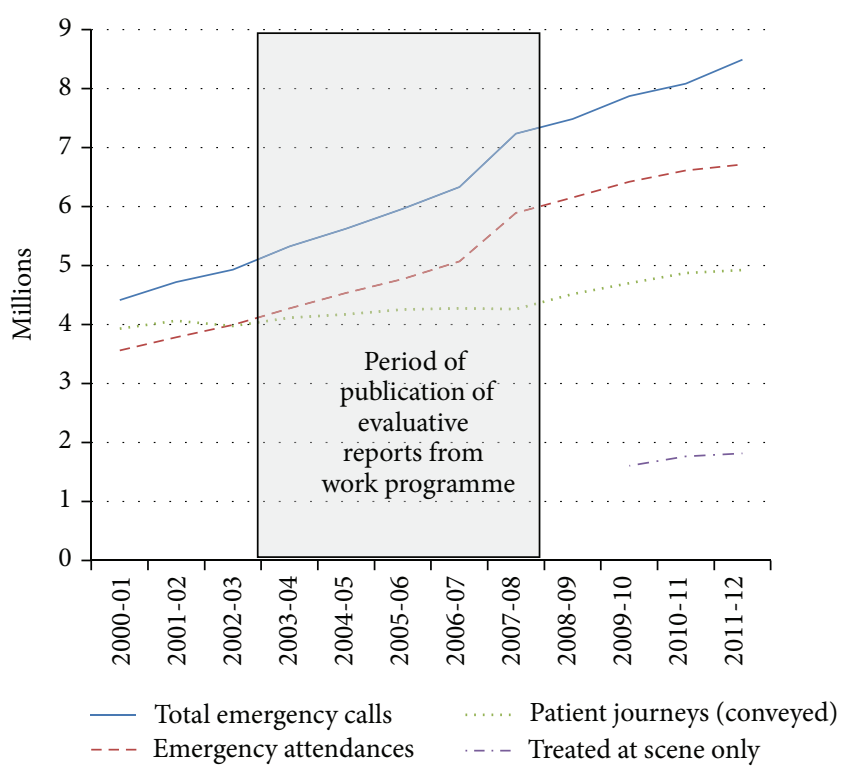

Figure 1: Ambulance Service Summary Statistics (a), England, 2000-2012.

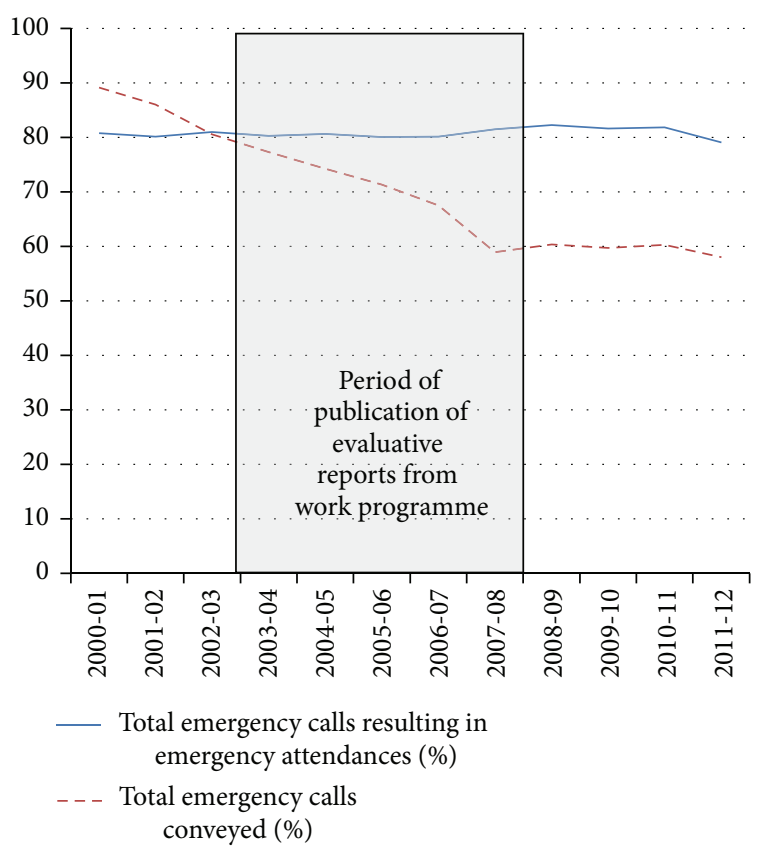

Figure 2: Ambulance Service Summary Statistics (b), England, 2000-2012.

approaches [35, 36]. In this under researched area, policy documents are often consensual rather than based on evidence and citation of underpinning research is rare.

In this paper we have described the scope, characteristics, and impact of a research programme in emergency prehospital care. For inferences about impact on practice we have had to rely on citations and ad hoc reports of service innovation alongside routine statistics related to emergency demand and treatment. Citations are recognised as a weak indicator of real impact [36]. Routine data are reliable but observational.
We are conscious that there are other potential causes for these changes. In an ideal or planned world with multiple indicators and well-defined interventions, the statistical technique of interrupted time series can draw stronger conclusions about cause and impact, as in the Respect trial [37, 38]. In the real world inferences about causation are more difficult.

4.3. Implications. Demonstration of impact of research is increasingly important in times when resources are scarce and competition is heavy. Research funders and researchers are under pressure to report impact but methods are underdeveloped. Policy and treatment guidelines often lack transparent underpinning research evidence. Measuring impact is our only way of capturing knowledge transfer from research evidence to patient care.

Against this setting we have attempted to set out the rationale, key features, and resulting impact on practice of a programme of research funded through the public purse in the UK. We argue that findings have been influential at national and international levels although we recognise that the rigour of methods for identifying and attributing impact is not as high as in the traditional "gold standard" RCT.

\section{References}

[1] D. Cooksey, A Review of UK Health Research Funding, HM Treasury, London, UK, 2006.

[2] L. A. Bero, R. Grilli, J. M. Grimshaw, E. Harvey, A. D. Oxman, and M. A. Thomson, "Getting research findings into practice. Closing the gap between research and practice: an overview of systematic reviews of interventions to promote the implementation of research findings," British Medical Journal, vol. 317, no. 7156, pp. 465-468, 1998.

[3] World Health Organization, World Report on Knowledge for Better Health: Strengthening Health Systems, World Health Organization, Geneva, Switzerland, 2004.

[4] S. R. Hanney, M. A. Gonzalez-Block, M. J. Buxton, and M. Kogan, "The utilisation of health research in policy-making: concepts, examples and method of assessment," Health Research Policy and Systems, vol. 1, no. 1, article 2, 2003.

[5] V. Bounes, E. Dehours, V. Houze-Cerfon, B. Valle, R. Lipton, and J. L. Ducasse, "Quality of publications in emergency medicine," American Journal of Emergency Medicine, vol. 31, no. 2, pp. 297-301, 2013.

[6] D. W. Spaite, E. A. Criss, T. D. Valenzuela, and J. Guisto, "Emergency medical service systems research: problems of the past, challenges of the future," Annals of Emergency Medicine, vol. 26, no. 2, pp. 146-152, 1995.

[7] Sheffield University, "Building the evidence base in pre hospital urgent and emergency care: a review of research evidence and priorities for future research," 2010.

[8] S. Brace and M. Cooke, "What are the priorities for prehospital research?” Journal of Paramedic Practice, vol. 2, no. 11, pp. 502504, 2010.

[9] H. Wrigley, S. George, H. Smith, H. Snooks, A. Glasper, and E. Thomas, "Trends in demand for emergency ambulance services in wiltshire over nine years: observational study," British Medical Journal, vol. 324, no. 7338, pp. 646-647, 2002. 
[10] H. Wrigley, H. Snooks, E. Thomas, H. Smith, A. Glasper, and S. George, "Epidemiology and demography of 999 ambulance calls: a review," Pre-Hospital Immediate Care, vol. 3, pp. 94-98, 1999.

[11] H. Snooks, H. Wrigley, S. George, E. Thomas, H. Smith, and A. Glasper, "Appropriateness of use of emergency ambulances," Journal of Accident and Emergency Medicine, vol. 15, no. 4, pp. 212-215, 1998.

[12] H. Snooks, S. Williams, R. Crouch, T. Foster, C. Hartley-Sharpe, and J. Dale, "NHS emergency response to 999 calls: alternatives for cases that are neither life threatening nor serious," British Medical Journal, vol. 325, no. 7359, pp. 330-333, 2002.

[13] H. A. Snooks, J. Dale, C. Hartley-Sharpe, and M. Halter, "Onscene alternatives for emergency ambulance crews attending patients who do not need to travel to the accident and emergency department: a review of the literature," Emergency Medicine Journal, vol. 21, no. 2, pp. 212-215, 2004.

[14] J. Dale, J. Higgins, S. Williams et al., "Computer assisted assessment and advice for "non-serious" 999 ambulance service callers: the potential impact on ambulance despatch," Emergency Medicine Journal, vol. 20, no. 2, pp. 178-183, 2003.

[15] J. Dale, S. Williams, T. Foster et al., "Safety of telephone consultation for "non-serious" emergency ambulance service patients," Quality and Safety in Health Care, vol. 13, no. 5, pp. 363-373, 2004.

[16] H. Snooks, T. Foster, and J. Nicholl, "Results of an evaluation of the effectiveness of triage and direct transportation to minor injuries units by ambulance crews," Emergency Medicine Journal, vol. 21, no. 1, pp. 105-111, 2004.

[17] H. Snooks, N. Kearsley, J. Dale, and M. Halter, "New models of care for 999 callers with conditions that are neither lifethreatening nor serious: results of a national survey," Pre-Hospital Immediate Care, vol. 4, pp. 180-182, 2000.

[18] H. Snooks, N. Kearsley, J. Dale, M. Halter, J. Redhead, and W. Y. Cheung, "Towards primary care for non-serious 999 callers: results of a controlled study of "Treat and Refer" for ambulance crews," Quality and Safety in Health Care, vol. 13, no. 6, pp. 435443, 2004.

[19] H. A. Snooks, N. Kearsley, J. Dale, M. Halter, J. Redhead, and J. Foster, "Gaps between policy, protocols and practice: a qualitative study of the views and practice of emergency ambulance staff concerning the care of patients with non-urgent needs," Quality and Safety in Health Care, vol. 14, no. 4, pp. 251-257, 2005.

[20] H. A. Snooks, M. Halter, J. C. T. Close, W. Cheung, F. Moore, and S. E. Roberts, "Emergency care of older people who fall: a missed opportunity," Quality and Safety in Health Care, vol. 15, no. 6, pp. 390-392, 2006.

[21] M. Halter, S. Vernon, H. Snooks et al., "Complexity of the decision-making process of ambulance staff for assessment and referral of older people who have fallen: a qualitative study," Emergency Medicine Journal, vol. 28, no. 1, pp. 44-50, 2011.

[22] J. Turner, H. Snooks, A. Youren et al., "The costs and benefits of managing some low-priority 999 ambulance calls by NHS Direct nurse advisers," Report for National Co-Ordinating Centre for NHS Service Delivery and Organisation R\&D, NCCSDO, 2006.

[23] A. Porter, H. Snooks, A. Youren et al., "Should I stay or should I go?' Deciding whether to go to hospital after a 999 call," Journal of Health Services Research and Policy, vol. 12, supplement 1, pp. S1-S1, 2007.
[24] A. Porter, H. Shooks, A. Youren et al., "“'Covering our backs”: ambulance crews' attitudes towards clinical documentation when emergency (999) patients are not conveyed to hospital," Emergency Medicine Journal, vol. 25, no. 5, pp. 292-295, 2008.

[25] S. Mason, E. Knowles, B. Colwell et al., "Effectiveness of paramedic practitioners in attending 999 calls from elderly people in the community: cluster randomised controlled trial," British Medical Journal, vol. 335, no. 7626, pp. 919-922, 2007.

[26] S. Mason, E. Knowles, J. Freeman, and H. Snooks, "Safety of paramedics with extended skills," Academic Emergency Medicine, vol. 15, no. 7, pp. 607-612, 2008.

[27] S. Dixon, S. Mason, E. Knowles et al., "Is it cost effective to introduce paramedic practitioners for older people to the ambulance service? Results of a cluster randomised controlled trial," Emergency Medicine Journal, vol. 26, no. 6, pp. 446-451, 2009.

[28] S. Thakore, E. A. McGugan, and W. Morrison, "Emergency ambulance dispatch: is there a case for triage?" Journal of the Royal Society of Medicine, vol. 95, no. 3, pp. 126-129, 2002.

[29] L. J. Morrison, L. M. Visentin, A. Kiss et al., "Validation of a rule for termination of resuscitation in out-of-hospital cardiac arrest," New England Journal of Medicine, vol. 355, no. 5, pp. 478487, 2006.

[30] Medical Research Council, "A Framework for development and evaluation of RCTs for Complex Interventions to Improve Health," 2000.

[31] P. Craig, P. Dieppe, S. Macintyre, S. Michie, I. Nazareth, and M. Petticrew, "Developing and evaluating complex interventions: the new Medical Research Council guidance," British Medical Journal, vol. 337, article a1655, 2008.

[32] Department of Health, Taking Healthcare To the Patient, Transforming NHS Ambulance Services, 2005.

[33] Welsh Assembly Government, A Guide to Good Practice: Emergency Care: Tools and Techniques to Enable the NHS and Social Services to Improve the Delivery of Health and Social Care, 2004.

[34] Health Workforce Australia, National Health Workforce Innovation and Reform Strategic Framework for Action 2011-2015, 2011.

[35] P. Davis and P. Howden-Chapman, "Translating research findings into health policy," Social Science and Medicine, vol. 43, no. 5, pp. 865-872, 1996.

[36] J. Lavis, S. Ross, C. McLeod, and A. Gildiner, "Measuring the impact of health research," Journal of Health Services Research and Policy, vol. 8, no. 3, pp. 165-170, 2003.

[37] RESPECT trial team, "Effectiveness of shared pharmaceutical care for older patients: RESPECT trial findings," British Journal of General Practice, vol. 60, no. 570, pp. e10-e19, 2010.

[38] RESPECT trial team, "Cost-effectiveness of shared pharmaceutical care for older patients: RESPECT trial findings," British Journal of General Practice, vol. 60, no. 570, pp. e20-e27, 2010. 


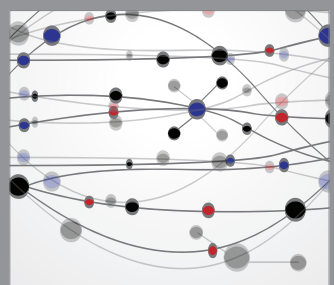

The Scientific World Journal
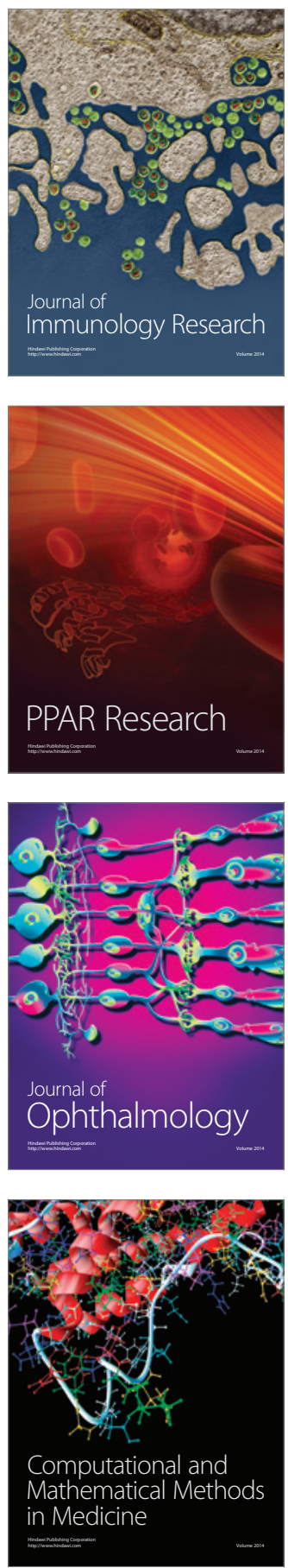

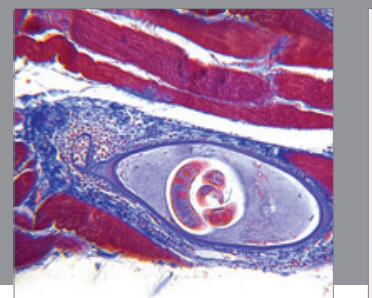

Gastroenterology

Research and Practice
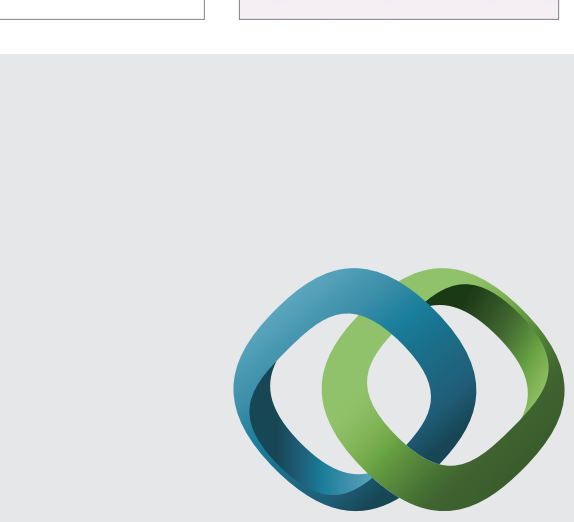

\section{Hindawi}

Submit your manuscripts at

http://www.hindawi.com
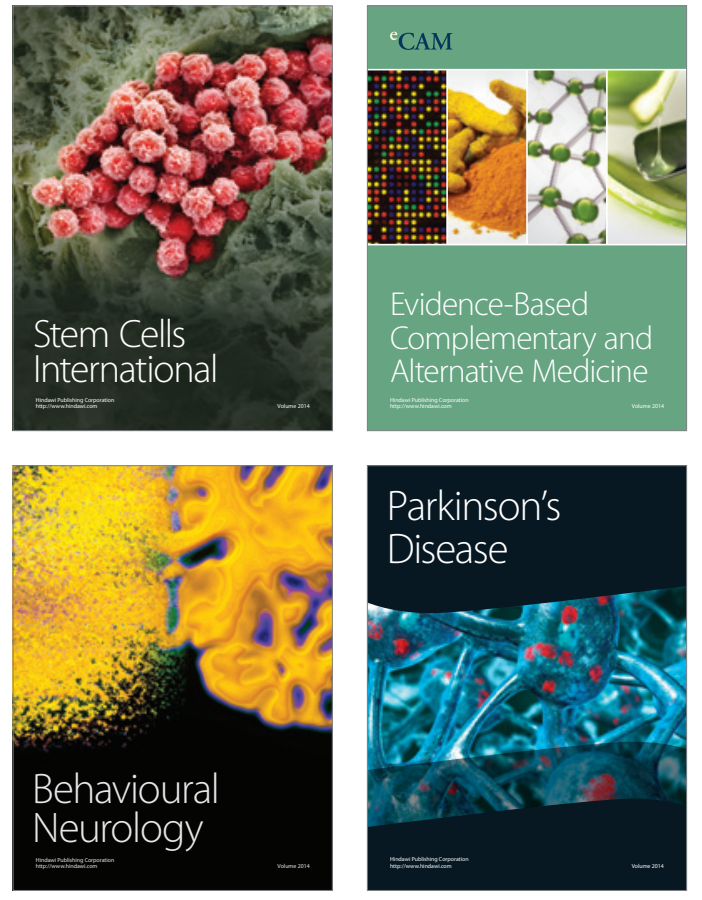
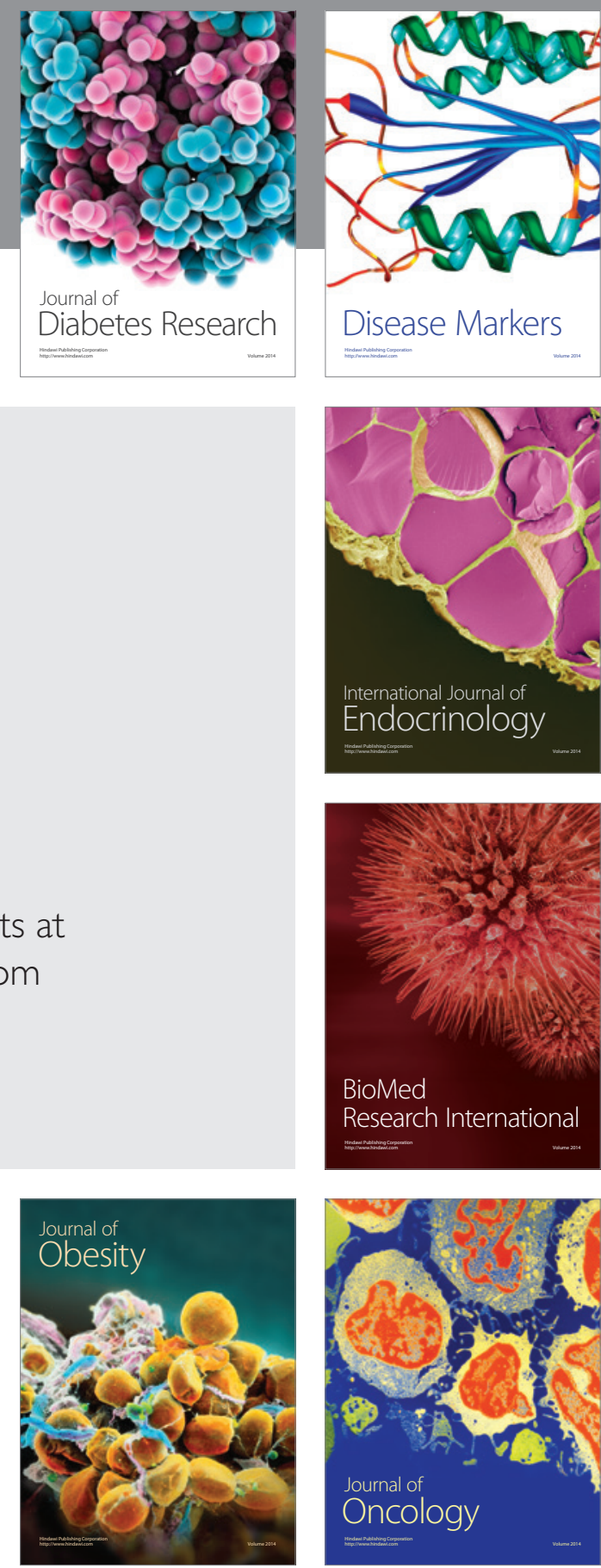

Disease Markers
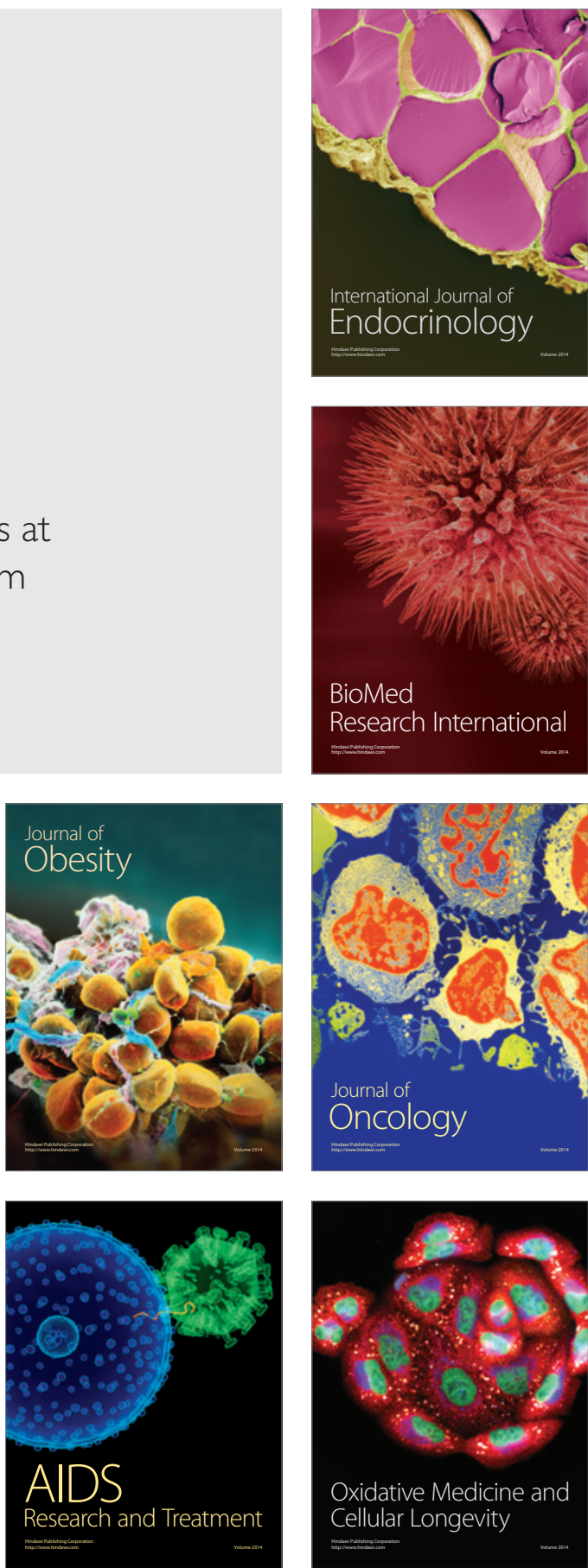